\title{
Image Analysis Studies for Phase Transitions of Ferroelectric Liquid Crystals
}

\section{S. Sreehari Sastry, ${ }^{1}$ B. Gowri Sankara Rao, ${ }^{1}$ K. B. Mahalakshmi, ${ }^{2}$ K. Mallika, ${ }^{1}$ C. Nageswara Rao, ${ }^{1}$ and Ha Sie Tiong ${ }^{3}$}

\footnotetext{
${ }^{1}$ Department of Physics, Acharya Nagarjuna University, Nagarjuna Nagar, Guntur 522510, India

${ }^{2}$ Department of Geology, Acharya Nagarjuna University, Nagarjuna Nagar, Guntur 522510, India

${ }^{3}$ Department of Chemical Science, Faculty of Science, Universiti Tunku Abdul Rahman, Jalan Universiti, Bandar Barat, 31900 Kampar, Malaysia
}

Correspondence should be addressed to S. Sreehari Sastry, sreeharisastry@yahoo.com

Received 5 September 2012; Accepted 24 September 2012

Academic Editors: C. Homes, R. Rossmanith, and S. Wang

Copyright () 2012 S. Sreehari Sastry et al. This is an open access article distributed under the Creative Commons Attribution License, which permits unrestricted use, distribution, and reproduction in any medium, provided the original work is properly cited.

\begin{abstract}
Phase transition temperatures of ferroelectric liquid crystals ((S)-(-)-2-methylbutyl $4^{\prime}$ - (4" -n-alkanoyloxybenzoyloxy) biphenyl-4carboxylates (where $n=16$ and 18)) are studied through two techniques of image analysis. One is a statistical method, applied to compute the statistical parameters from the textures of each sample and the other, computation of Legendre moments being applied as image moment analysis, both of which are considered as a function of temperature. The textures of the samples are recorded with the polarizing optical microscope (POM) attached to the hot stage and high resolution camera. The phase transition temperatures of samples are inferred by the abrupt changes in the computed parameter values. The results obtained from the present methodology are in good agreement with those published in earlier literature done by the different techniques, like differential scanning calorimetry (DSC).
\end{abstract}

\section{Introduction}

The studies on phase transitions in thermotropic liquid crystal have seen considerable progress since 1980 [1]. With the availability of high resolution and sensitive experimental techniques, it could be made easy to record precisely the transformations that occur during mesophase changes. Identification of transition phases and transition temperatures is the vital aspect to characterize the properties of liquid crystal compounds. Besides, the ordering in phase transitions, namely, first-order/second-order phases, is crucial in current liquid crystal field studies [2-4]. With its wide applications in the use of optical switches, light valves, display and storage devices, and other electrooptic devices, in particular the study on the ferroelectric liquid crystals (FLCs) has generated much interest for synthesizing and characterizing the new FLCs [5-9]. Having these objectives, the present attempt is aimed to study the transition temperatures of the two synthesized ferroelectric liquid crystals ((S)-(-)-2-methylbutyl $4^{\prime}-\left(4^{\prime \prime}-n\right.$-alkanoyloxybenzoyloxy) biphenyl-4-carboxylates (where $n=16$ and 18)) through the image analysis techniques, statistical and image moments approaches applied to the microscopic textures of FLCs. The results of the study are compared with those of published earlier studies [10]. Also this kind of work was done for room temperature liquid crystals, cholesteric liquid crystals, and for discotic liquid crystals [11-15].

\section{Experimental}

The liquid crystals of chiral esters (S)-(-)-2-methylbutyl $4^{\prime}-\left(4^{\prime \prime}-n\right.$-alkanoyloxybenzoyloxy) biphenyl-4-carboxylates where $n=16$ and 18 having different alkyl chain lengths are synthesized [10] and used. The ITO coated homogeneous (planar) cells having an area of $5 \mathrm{~mm} \times 5 \mathrm{~mm}$ with $8.7 \mu \mathrm{m}$ 
spacing (tolerance is $\pm 0.2 \mu \mathrm{m}$ ) are obtained from Instec Inc., USA. Homogeneous (planar) alignments are obtained in the sandwich cell with surfaces, which were treated with a rubbing method. The chiral ester mesogens are injected into liquid-crystal cells through capillary action on heating the sample to the isotropic state. Different phase transitions can be observed with the naked eye at the time of cooling the liquid crystal cell after injecting the samples and good alignment is achieved by cooling down the cell very slowly. The flowing direction of the liquid crystal during cell filling is found to influence the molecular alignment in the resultant liquid crystal layer. Obviously the appearance will be strictly controlled by the nature of the alignment surface in the cells that are used. Meopta polarizing optical microscope is used to record the respective transformation phases of sample textures simultaneously and instantaneously as a function of temperature with its auxiliary attachments, the "Hot Stage" described by Gray [16] and "the High Resolution Camera."

A high resolution Canon EOS Digital REBEL XS/ EOS1000D camera with 10.10 megapixel image sensor is used for recording the observations. The colour image detected by the camera has a resolution of $2816 \times 1880$ pixels which represents the 24 bit true colour pixel tone that ranges and the intensity values from 0 to 255 in $R, G$, and $B$ colours [17]. In the present work, the translated gray scale image is used for analysis. The size of images are selected to be 256. The program has been coded using MATLAB software (implemented on P5 $1.6 \mathrm{GHz}$ with $2 \mathrm{~GB}$ RAM computer) for analysis of textures which is an efficient tool for computational analysis $[18,19]$.

2.1. Theory. Image analysis is the method used to extract the meaningful information from images by means of applying computational techniques and algorithms on image data. The statistical computations and measurements carried out in the image analysis are based on gray level intensities of image pixels. The statistical methods are applied to extract the local features from the spatial distribution of gray levels of image and to derive a set of statistical parameters from the distribution of the local features [20, 21]. Depending on the number of pixels defining the local features, the statistical parameters are further classified into the first-order (one pixel), the second-order (two pixel), and higher-order statistics. The basic difference is that first-order statisticsmean, standard deviation, skewness, and kurtosis-estimate the properties based on individual pixel values ignoring the spatial interactions between image pixels; whereas the second-order statistics-entropy, energy, homogeneity, and correlation-are being used to calculate the properties based on two pixel values occurring adjacent to each other at specific locations [22].

Image moment is another statistical measure, in which Legendre moments are used to analyze the liquid crystal texture images. Legendre moments infer the image with minimum information redundancy. Legendre moments of the textures are obtained by mapping the image intensity values on Legendre polynomial functions $[23,24]$. Since it is very sensitive, the presence of smooth transitions and the transition temperatures are efficiently determined.

An image or texture $I(i, j)$ is of size $m$-by-nis a two dimensional function composed of $m$ pixels in the vertical direction and $n$ pixels in the horizontal direction, $i, j$ are horizontal and vertical coordinates of the image. The total number of pixels in the image is $m^{*} n=N, 0 \leq i \leq m$, $0 \leq j \leq n$. The defined statistical parameters are explained below.

\subsubsection{Computation of Statistical Parameters}

(i) Mean. The mean $(\mu)$ is defined as the average level of intensity values of the image or texture $I(i, j)$ :

$$
\mu=\frac{1}{N} \sum_{i=1}^{m} \sum_{j=1}^{n} I(i, j)
$$

(ii) Standard Deviation. Standard deviation of the image is defined as the square root of the variance. Variance defines the variation of intensity values around the mean intensity. If the variance value is closer to the mean, the standard deviation is less:

$$
S=\sqrt{\frac{\sum_{i=1}^{m} \sum_{j=1}^{n}(I(i, j)-\mu)^{2}}{N-1}} .
$$

(iii) Skewness. It defines the symmetry around the mean intensity value:

$$
\text { Skewness }=\frac{\left(\sum_{i=1}^{m} \sum_{j=1}^{n} I(i, j)-\mu\right)^{3}}{(N-1)^{*} S^{3}},
$$

where $S$ is standard deviation of the image.

(iv) Kurtosis. Kurtosis is a measure of peakedness, that is, whether the intensity values are peaked or flat around the mean intensity value:

$$
\text { Kurtosis }=\frac{\left(\sum_{i=1}^{m} \sum_{j=1}^{n} I(i, j)-\mu\right)^{4}}{(N-1)^{*} S^{4}} .
$$

The second-order statistical parameters are calculated from the gray level cooccurrence matrix (GLCM) of the texture [20-22].

(v) Entropy. Entropy is the measure of randomness of the gray levels in an image. The entropy of an image $I$ is calculated by finding the probability $P$ of a particular gray level value found in that image:

$$
\text { Entropy }=-\sum_{i=1}^{m} \sum_{j=1}^{n} P_{(i, j)} \log \left(P_{(i, j)}\right) .
$$

(vi) Energy. Energy measures the textural uniformity, that is, pixel pair repetitions. Maximum energy of the texture 


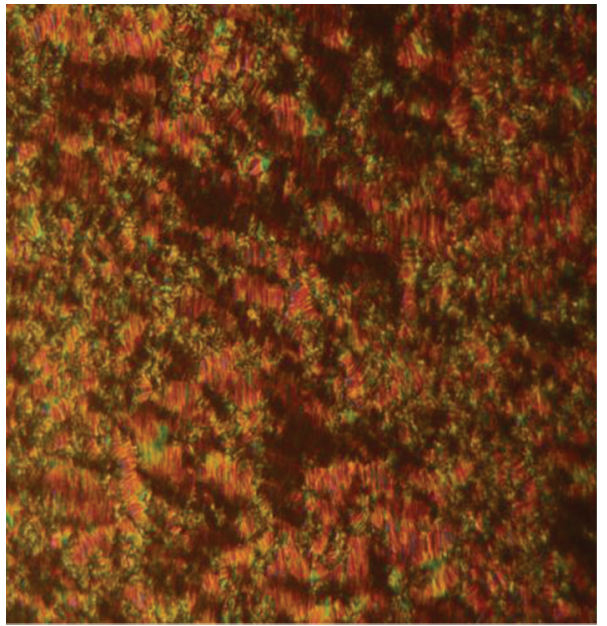

(a)

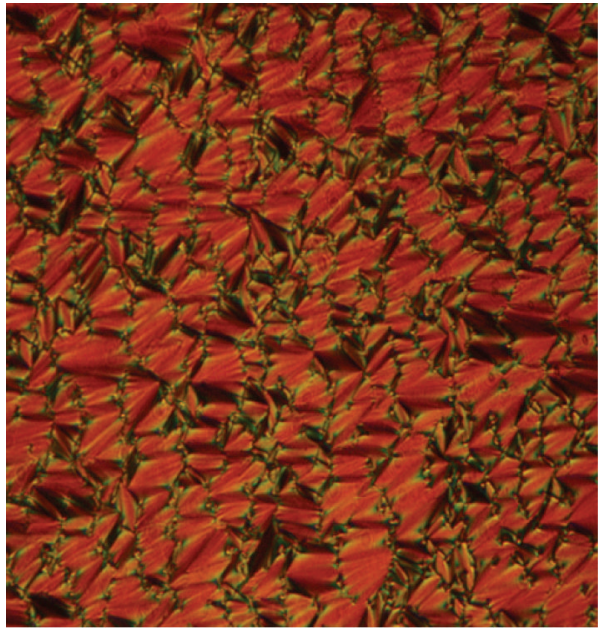

(c)

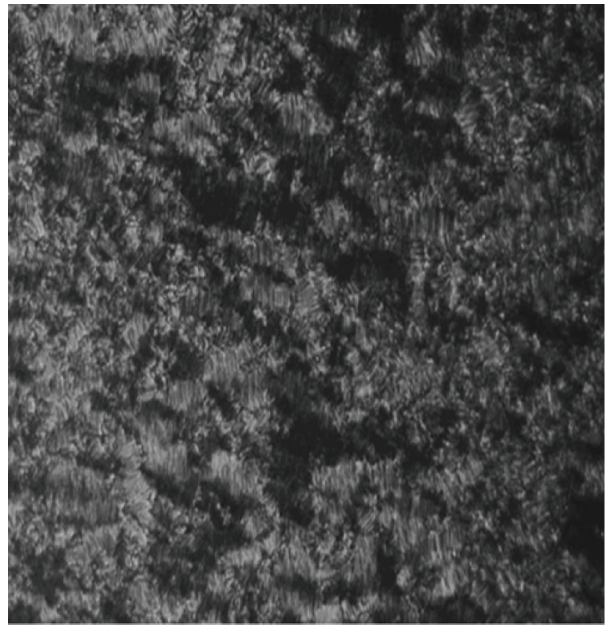

(b)

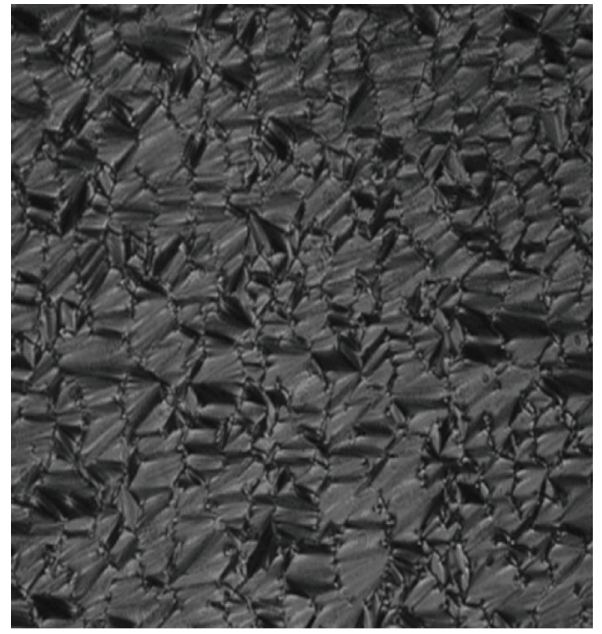

(d)

Figure 1: Optical textures of ((S)-(-)-2-methylbutyl $4^{\prime}-\left(4^{\prime \prime}\right.$-n-alkanoyloxybenzoyloxy) biphenyl-4-carboxylates (where $\left.n=16\right)$ ) liquid crystal. (a) SmecticC* phase, (b) smecticC* phase (grey colour), (c) smecticA phase, and (d) smecticA phase (grey colour).

or image occurs when the gray level distribution is either constant or a periodically uniform:

$$
\text { Energy }=\sum_{i=1}^{m} \sum_{j=1}^{n}(\operatorname{GLCM}(i, j))^{2} .
$$

(vii) Homogeneity. Homogeneity measures the closeness of the distribution of values in the GLCM. Homogeneous texture will have only a limited range of gray levels, giving a GLCM only a few values but relatively high probability $(P(i, j))$ for the GLCM values. Thus the sum of squares will be high. Energy and homogeneity are similar measures, the only difference is that energy considers the elements of the GLCM and homogeneity considers the probability of GLCM values:

$$
\text { Homogeneity }=\sum_{i=1}^{m} \sum_{j=1}^{n}(P(i, j))^{2} \text {. }
$$

(viii) Correlation. Correlation is a measure of gray level linear dependence between the pixels at specified positions relative to each other:

$$
\text { Correlation }=\sum_{i=1}^{m} \sum_{j=1}^{n} \frac{\{i \times j\} \times P(i, j)-\left\{\mu_{x} \times \mu_{y}\right\}}{\sigma_{x} \times \sigma_{y}},
$$

where $\mu_{x}, \mu_{y}$ and $\sigma_{x}, \sigma_{y}$ are the mean and standard deviations of probability matrix $P$ along row-wise $\left(P_{x}\right)$ and column-wise $\left(P_{y}\right)$.

All the defined parameters are extremely sensitive to changes in the textural features of liquid crystals as a function of temperature [11-15]. But, these statistical parameters are unable to express the spatial relationships of different local textural patterns in the image and are not robust in the presence of image noise.

2.1.2. Computation of Legendre Moments. Legendre moment or Legendre polynomial is a mathematical concept has been 


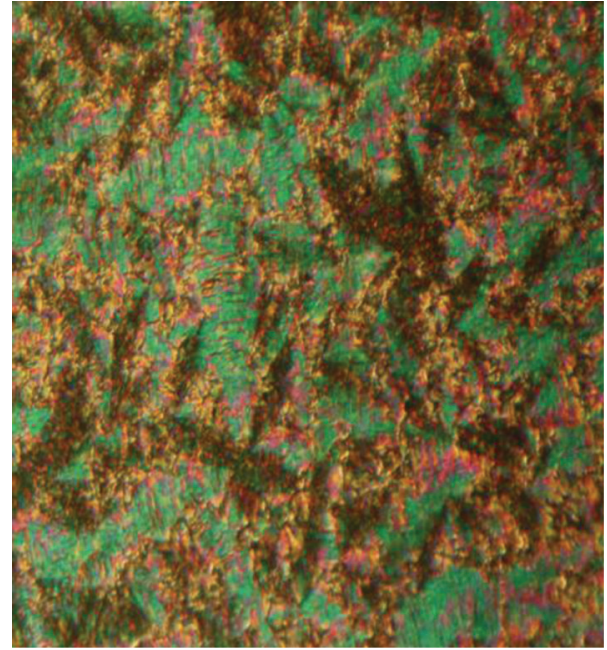

(a)

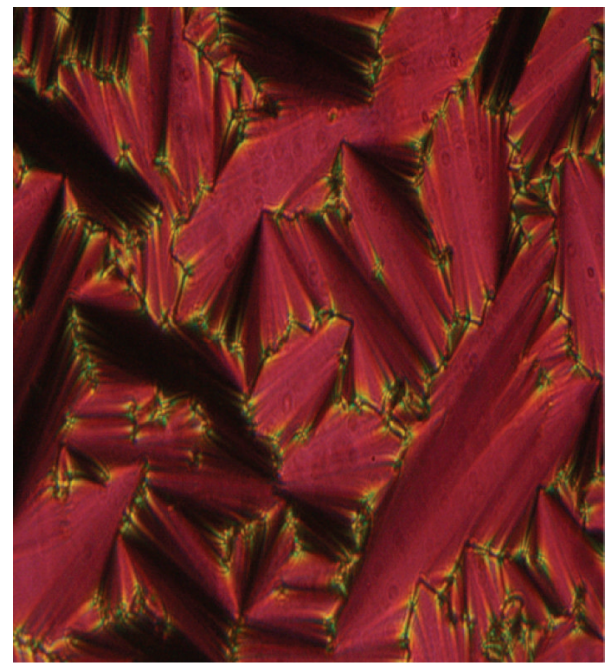

(c)

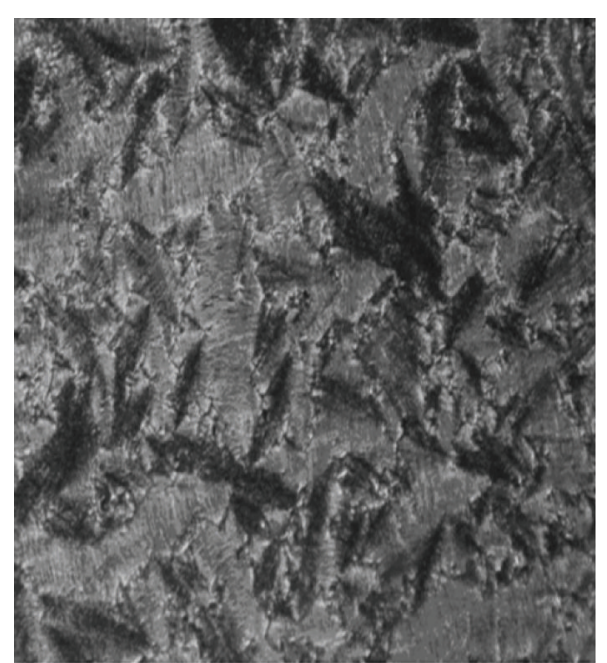

(b)

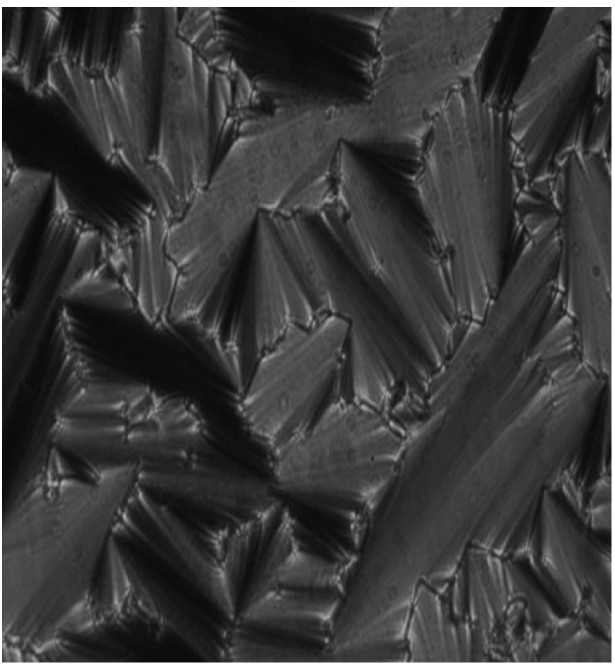

(d)

FIGURE 2: Optical textures of ((S)-(-)-2-methylbutyl $4^{\prime}-\left(4^{\prime \prime}-n\right.$-alkanoyloxybenzoyloxy) biphenyl-4-carboxylates (where $\left.n=18\right)$ liquid crystal. (a) Smectic ${ }^{*}$ phase, (b) smecticC* phase (grey colour), (c) smecticA phase, and (d) smecticA phase (grey colour).

TABLE 1: CPU elapsed time for different moment orders.

\begin{tabular}{lcc}
\hline Image size & Moment order & CPU elapsed time (in seconds) \\
\hline \multirow{3}{*}{512} & 3 & 6.4184 \\
& 10 & 46.4834 \\
& 20 & 165.7872 \\
& 30 & 362.1196 \\
256 & 3 & 1.8975 \\
& 10 & 11.7815 \\
& 20 & 41.5352 \\
& 30 & 88.9957 \\
\hline
\end{tabular}

used to describe the objects of an image. Describing images with moments (weighted average of the intensity values) instead of other image features gives the attractive interpretation about images. Legendre moments are global features which extract useful information from the images for the purpose of analysis. Legendre moments of the textures are obtained by mapping or projecting the image intensity values on Legendre polynomial. It is a computational analysis to determine the transition temperatures of liquid crystals and is very sensitive to determine the occurrence of smooth transitions. The advantage of using Legendre moments is that these are invariant with respect to the transformations of scale, translation, and rotation. Legendre moments identify the changes in features of the liquid crystal texture from the initial state (solid) to the final state (isotropic) with respect to the temperature. Theory related to the generation of Legendre polynomial basis, computation of Legendre moments of the textures and drawing the Legendre moment plots of the textures as a function of temperature which gives the phase transition temperatures of liquid crystals are briefly explained in [11, 24-26]. 


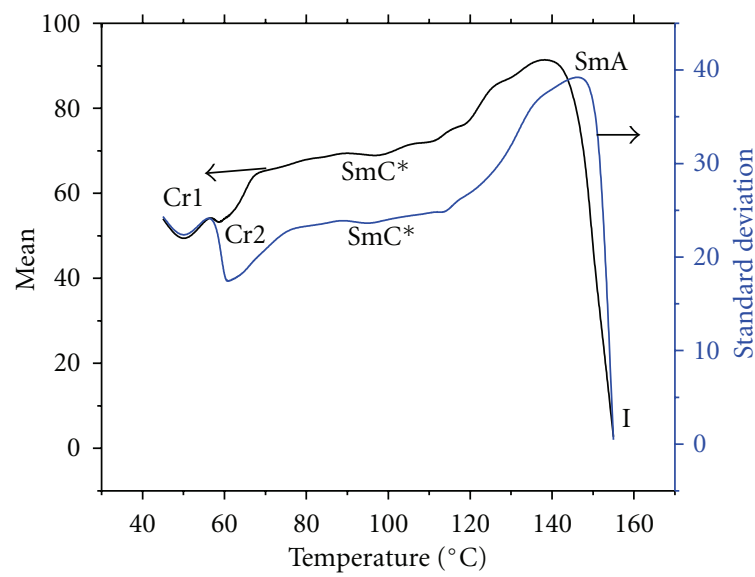

(a)

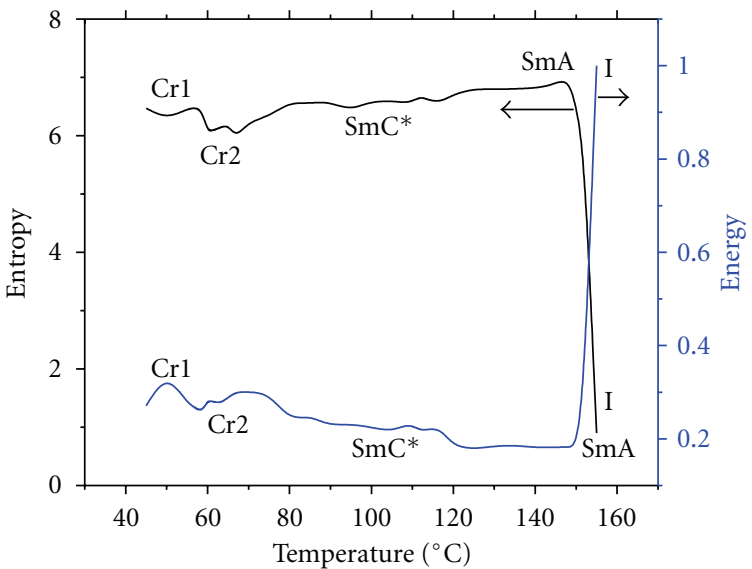

(c)

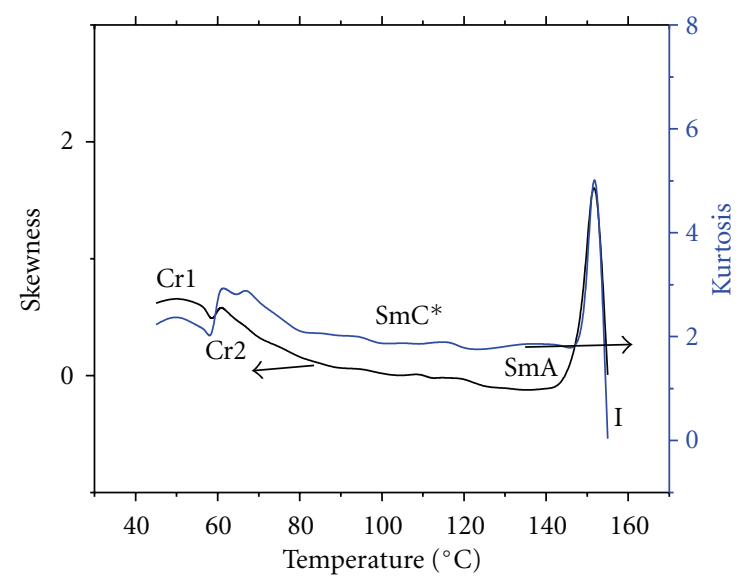

(b)

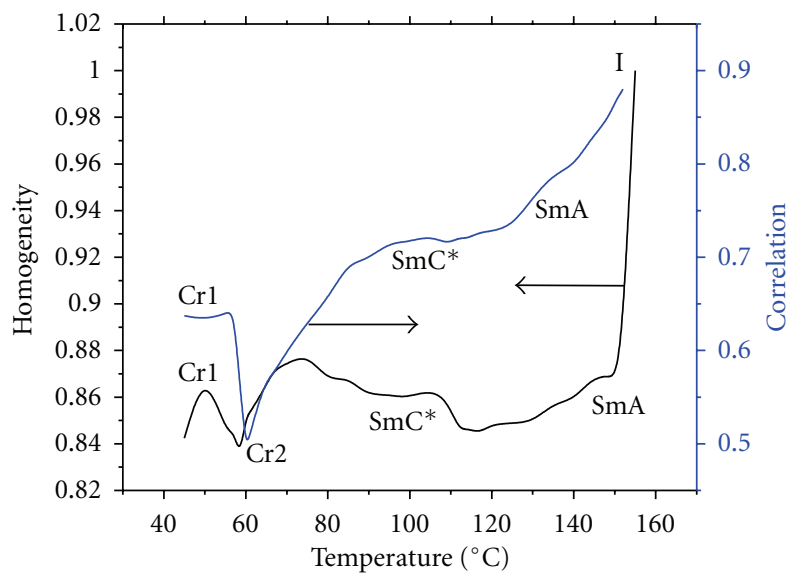

(d)

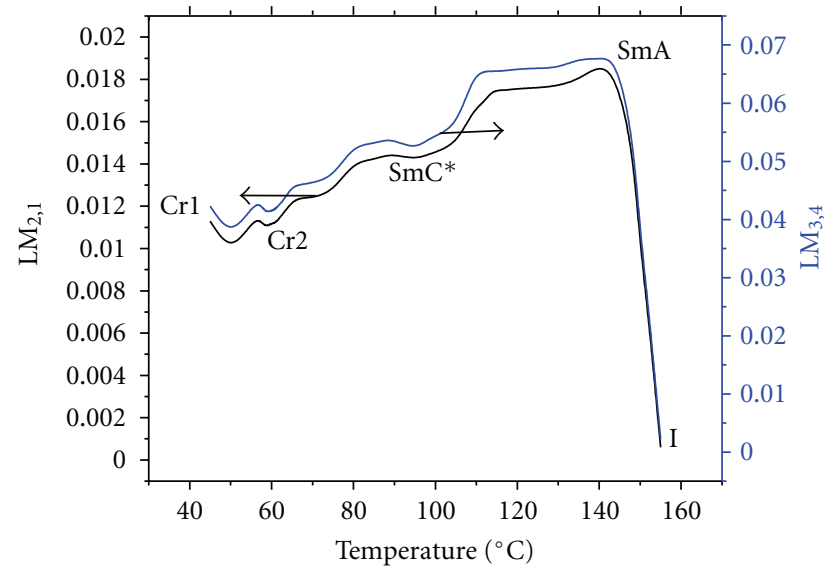

(e)

Figure 3: Computed parameters from the textures of (S)-(-)-2-methylbutyl $4^{\prime}-\left(4^{\prime \prime}-n\right.$-alkanoyloxybenzoyloxy) biphenyl-4-carboxylates (where $n=16$ ) as a function of temperature where the phase transitions are indicated. (a), (b), for first-order statistical parameters, (c), (d) for second-order statistical parameters, and (e) for Legendre moments.

\section{Results and Discussion}

Textures of the ((S)-(-)-2-methylbutyl 4' - (4' - $n$-al-kanoyloxybenzoyloxy) biphenyl-4-carboxylates (where $n=16$ and 18)) liquid crystals are recorded using POM as a function of temperature. The textures of the samples are recorded at the heating/cooling rate of $0.2^{\circ} \mathrm{C}$ per minute from the solid phase of the sample to isotropic phase (I) on heating and reverse on cooling from isotropic phase to solid phase.

On heating and cooling, the samples exhibited the nontilted (smecticA) and tilted (smectic $\left.{ }^{*}\right)$ smectic phases. These FLCs undergoes a phase transitions from crystalline 


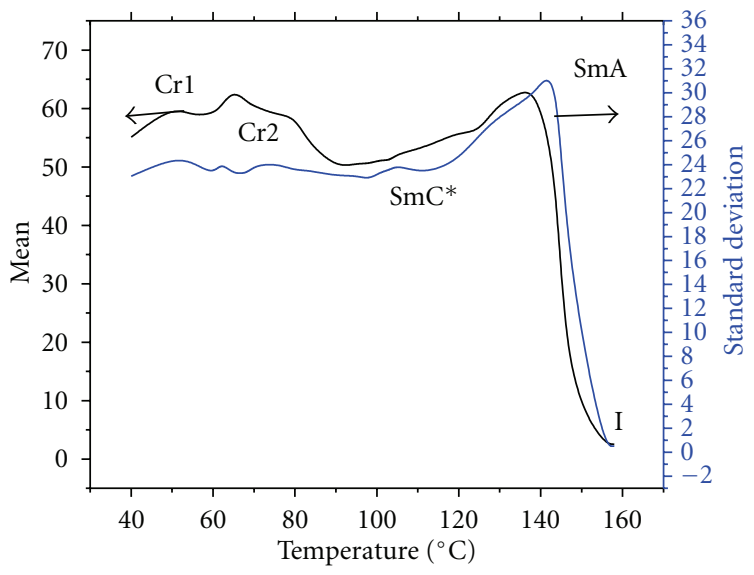

(a)

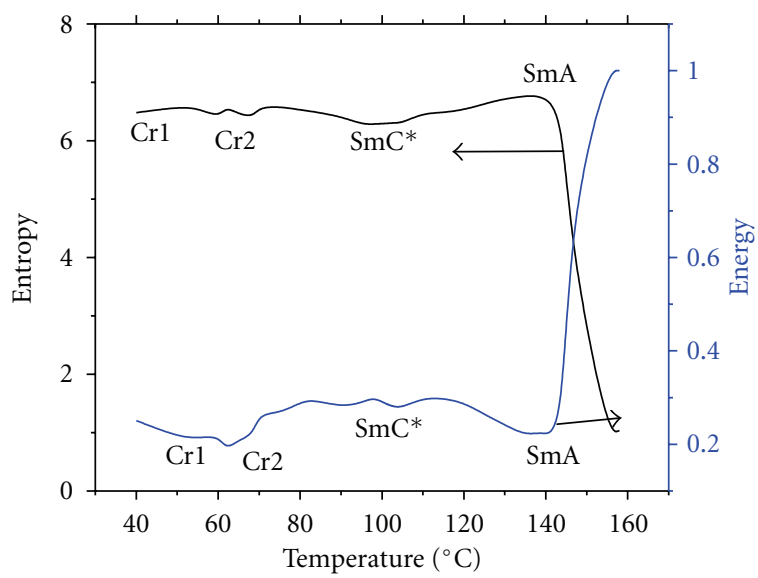

(c)

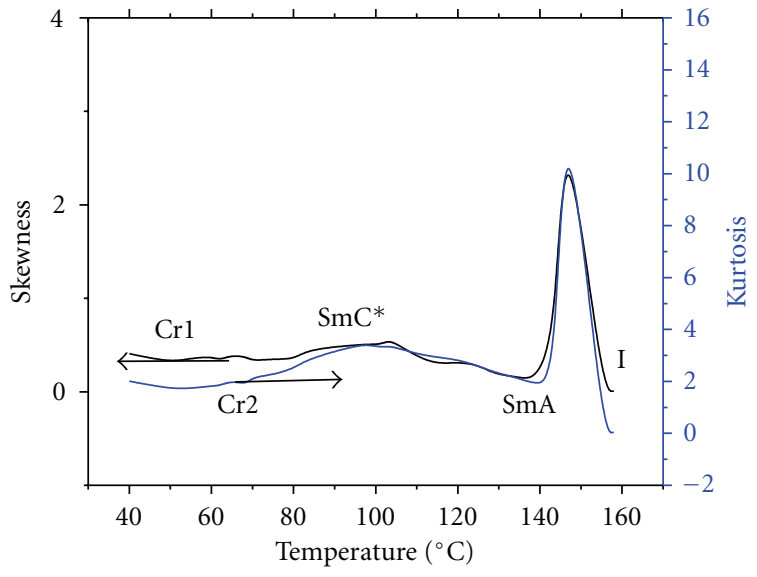

(b)

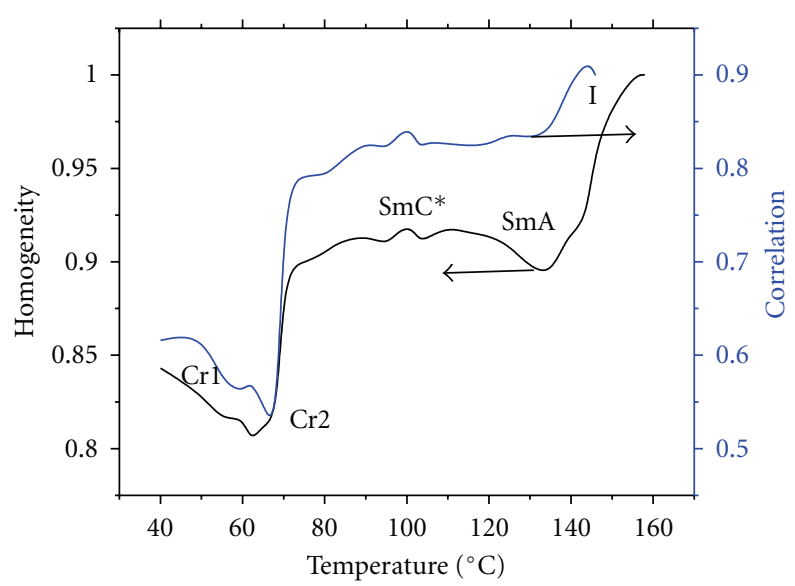

(d)

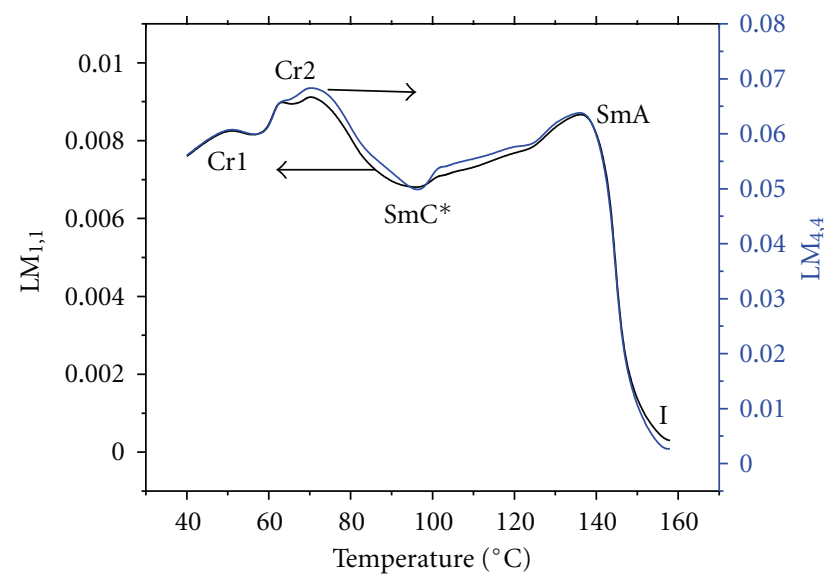

(e)

FIGURE 4: Computed parameters from the textures of (S)-(-)-2-methylbutyl $4^{\prime}-\left(4^{\prime \prime}-n\right.$-alkanoyloxybenzoyloxy) biphenyl-4-carboxylates (where $n=18$ ) as a function of temperature where the phase transitions are indicated. (a), (b) for first-order statistical parameters, (c), (d) for second-order statistical parameters, and (e) for Legendre moments.

phase to the isotropic phase via smectic $C^{*}$, smecticA, that is, Cr-smecticC*, smecticC*-smecticA, smecticA-Isotropic (I) on heating and reverse trend in cooling. But, on cooling with in the crystal phase given FLCs exhibits the two subphases of crystal 1 ( $\mathrm{Cr} 1)$, crystal 2 (Cr2), that is, I-smecticA,
smecticA-smecticC*, smecticC*-Cr2, Cr2-Cr1. Textures of the samples are shown in Figures 1 and 2.

Results obtained from the statistical techniques are shown in the Figures 3 and 4 . The experiment was done for both heating and cooling cycles. To avoid the paramorphic 
TAble 2: Transition temperatures (in $\left.{ }^{\circ} \mathrm{C}\right)$ of chiral ester smectogens (S)-(-)-2-methylbutyl $4^{\prime}-\left(4^{\prime \prime}-n\right.$-alkanoyloxybenzoyloxy) biphenyl-4carboxylates.

\begin{tabular}{lll}
\hline Compounds & Present method & Literature [10] \\
\hline$n=16$ & Heating: Cr 62 SmC* 112 SmA 153 I & Cr 64 SmC* 112.4 SmA 153.2 I \\
& Cooling: I 152.5 SmA 101.5 SmC*60 Cr2 51 Cr1 & I 152.8 SmA 102.4 SmC* 60.7 Cr2 152.4 Cr1 \\
$n=18$ & Heating: Cr 74.6 SmC* 113 SmA 152 I & Cr 73.3 SmC* 112 SmA 152.4 I \\
& Cooling: I 150.1 SmA 103 SmC* 63 Cr2 60 Cr1 & I 151.8 SmA 104.5 SmC* 68.5 Cr2 62.7 Cr1 \\
\hline
\end{tabular}

defects, as a representative case and the plots are drawn for cooling cycles. The nature of behavior for computed parameters curves is different for different compounds of FLCs.

The significant variations in the computed parameter values shown in Figures 3 and 4 are due to the fact that transition in phases caused the changes in the textural features. During both heating or on cooling cycles, changes in the textural features would take place as a function of temperature. These are more identifiable at the transition which is indication of phase transition. Any small changes in the textural features bring variations in the transmitted intensity or gray values, which are useful to compute the necessary parameters. Therefore, the distinctive and abrupt changes as observed in the parameters curve at temperature corresponding to the transition temperature of samples have ensured that the present methodology is a very sensitive method for identification of phase transitions of liquid crystals [11-15].

In DSC, changes in enthalpy values for the secondorder transitions are relatively small and are not observable like first-order transitions $[4,8]$. This was shown in Figure 5 drawn for the ferroelectric liquid crystal (S)-(-)2-methylbutyl $4^{\prime}$-( $4^{\prime \prime}-n$-alkanoyloxybenzoyloxy) biphenyl4-carboxylates (where $n=16$ ). Like in DSC, the first-order statistical parameters skewness, kurtosis and the secondorder statistical parameters energy, correlation did not show much variation in the values at the transitions of some phases. But this is true for some samples and is apparent in Figures 3 and 4. For the transition from SmA-SmC* the first-order parameters (skewness, kurtosis) and second-order parameters (entropy and correlation) did not show abrupt changes in values. The parameter values computed for the textures at respective temperatures are correlated with each other and their differences did not contribute to show abrupt changes. But, these features showed abrupt changes for the transition to I-liquid crystal phase or liquid crystal phase-I of any liquid crystal and are shown in Figures 3(b) and 4(b). This is because of large differences in mean intensity values reported in heating and cooling cycles while reaching to the isotropic state and from isotropic to liquid crystal state, respectively. Despite, the first-order statistical parameters mean, standard deviation, second-order statistical parameters energy, homogeneity, and Legendre moments showed abrupt changes at the transition. Compared to the statistical parameters, image moments, Legendre moments yield the best results for any compounds. This is due to the fact that image moments are global features and robust in front of

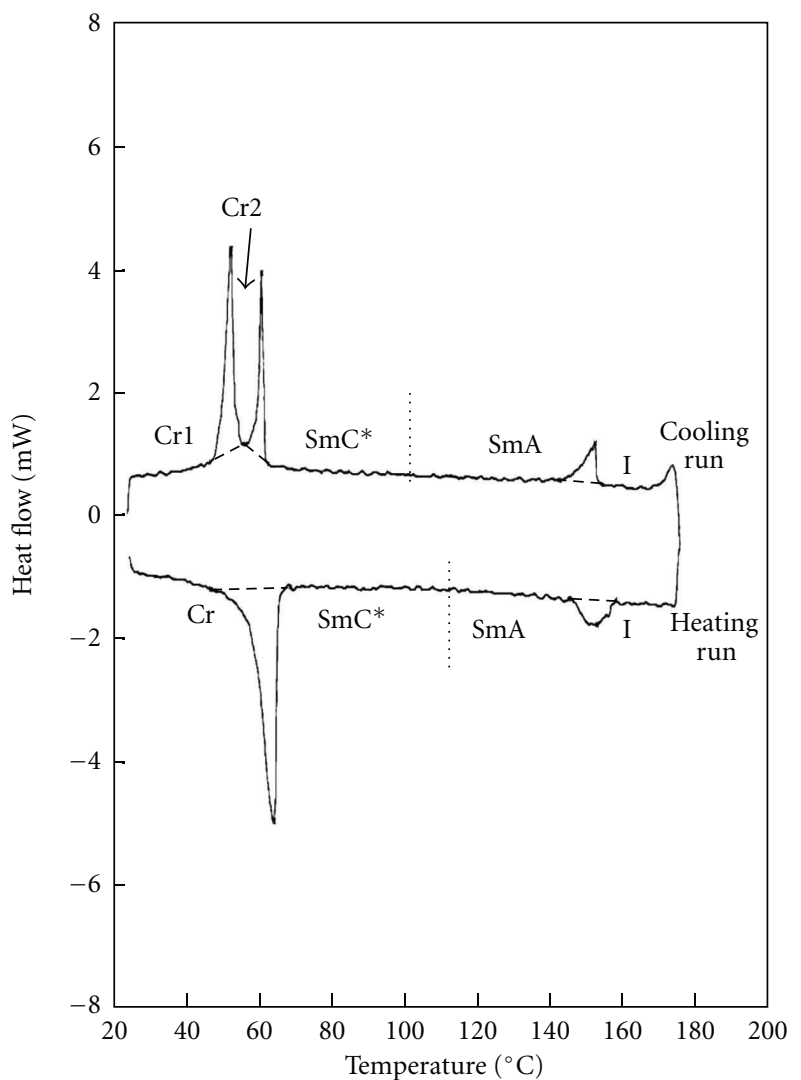

FIGURE 5: DSC graph of ferroelectric liquid crystal (S)-(-)-2methylbutyl $4^{\prime}-\left(4^{\prime \prime}-n\right.$-alkanoyloxybenzoyloxy) biphenyl-4-carboxylates (where $n=16$ ).

noise, provided the good results with minimum amount of information redundancy. On the context of applications, the Legendre moments will calculate for high orders. But the difficulty in the use of moments is its high computational complexity, especially when a higher order of moments is used $[25,26]$. Table 1 detailed the moment order or the polynomial order $3,10,20,30$ at image of size 256, 512, the computational time, or CPU elapsed time required to calculate the Legendre moments of the textures. Both lowerorder moments and higher-order moments are useful to investigate the phase transitions of liquid crystals. The phase transition temperatures obtained from the present study are shown in Table 2 along with the values in the literature [10] for comparison. 


\section{Conclusion}

Phase transition temperatures of the FLCs are computed through image analysis technique. Techniques mentioned in literature are relates to heat energy, heat flow, and enthalpy values, but this technique depends on gray level intensity values of the textures and their changes. The result obtained from the present methodology is compared with the other standard technique, like DSC, which is reasonably in good agreement.

\section{Abbreviations}

$\begin{array}{ll}\text { Cr: } & \text { Crystal } \\ \text { Cr1: } & \text { Crystal 1 } \\ \text { Cr2: } & \text { Crystal 2 } \\ \text { SmC: } & \text { SmecticC } \\ \text { SmA: } & \text { SmecticA } \\ \text { I: } & \text { Isotropic. }\end{array}$

\section{Acknowledgments}

The authors gratefully acknowledge the Project no. 3412/2008 (SR), dated 30-12-2008 of UGC, UGC DRS LEVEL III Program no. F.530/1/DRS/2009 (SAP-I), dated 09-022009, and DST FIST Program no. DST/FST/PSI-002/2011, dated 20-12-2011, New Delhi, also the Department of Physics, ANU for providing financial assistance. Ha Sie Tiong would like to thank Universiti Tunku Abdul Rahman for UTAR Research Fund (6200H10).

\section{References}

[1] D. Demus, J. Goodby, G. W. Gray, H. W. Spiess, and V. Vill, Hand Book of Liquid Crystals, Wiley-VCH, Singapore, 1998.

[2] S. Singh, Liquid Crystals Fundamentals, World Scientific, Singapore, 2002.

[3] D. Demus, J. Goodby, G. W. Gray, H. W. Spiess, and V. Vill, Physical Properties of Liquid Crystals, Wiley-VCH, Singapore, 1999.

[4] S. Chandra Sekhar, Liquid Crystals, Cambridge University Press, New York, NY, USA, 1992.

[5] P. Oswald and P. Pieranski, The Liquid Crystal Books SeriesSmectic and Columnar Liquid Crystals_Concepts and Physical Properties Illustrated by Experiments, CRC Press, Taylor \& Francis Group, London, UK, 2006.

[6] I.-C. Khoo, Liquid Crystals, Wiley-Interscience, John Wiley \& Sons, Hoboken, NJ, USA, 2007.

[7] R. B. Meyer, "Ferroelectric liquid crystals: a review," Molecular Crystals and Liquid Crystals, vol. 40, no. 1-4, pp. 33-48, 1977.

[8] R. B. Meyer, L. Liebert, L. Strzelecki, and P. Keller, "Ferroelectric liquid crystals : a review," Journal of Physical Chemistry Letters, vol. 36, pp. 69-71, 1975.

[9] J. W. Goodby, E. Chin, J. M. Geary, J. S. Patel, and P. L. Finn, "The ferroelectric and liquid-crystalline properties of some chiral alkyl 4-n-alkanoyloxybiphenyl-4' -carboxylates," Journal of the Chemical Society, Faraday Transactions 1, vol. 83, no. 11, pp. 3429-3446, 1987.
[10] S.-T. Ha, G. Y. Yeap, and P. L. Boey, "Synthesis and smectogenic A and $C^{*}$ properties of $(\mathrm{S})-(-)-2$-methylbutyl $4^{\prime}-\left(4^{\prime \prime}-\right.$ n-alkanoyloxybenzoyloxy) biphenyl-4-carboxylates," International Journal of Physical Sciences, vol. 5, no. 3, pp. 182-191, 2010.

[11] S. S. Sastry, K. Mallika, B. Gowri Sankara Rao, H. S. Tiong, and S. Lakshminarayana, "Novel approach to study liquid crystal phase transitions using Legendre moments," Phase Transitions, vol. 85, no. 5, pp. 735-749, 2012.

[12] S. S. Sastry, K. Mallika, B. Gowri Sankararao, H. S. Tiong, and S. Lakshminarayana, "Identification of phase transition temperatures by statistical image analysis," Liquid-Crystal, vol. 39, no. 6, pp. 695-700, 2012.

[13] B. Montrucchio, A. Sparavigna, and A. Strigazzi, "A new image processing method for enhancing the detection sensitivity of smooth transitions in liquid crystals," Liquid Crystals, vol. 24, no. 6, pp. 841-852, 1998.

[14] A. Sparavigna, A. Mello, and B. Montrucchio, "Texture transitions in the liquid crystalline alkyloxybenzoic acid 6OBAC," Phase Transitions, vol. 79, no. 4-5, pp. 293-303, 2006.

[15] J. Eccher, A. R. Sampaio, R. C. Viscovini et al., "Image processing as a tool for phase transitions identification," Journal of Molecular Liquids, vol. 153, no. 2-3, pp. 162-166, 2010.

[16] W. Gray, Molecular Structure and the Properties of Liquid Crystals, Academic Press, New York, NY, USA, 1962.

[17] Canon REBEL XS EOS, "1000D Review and Microscope Application".

[18] R. C. Gonzalez, R. E. Woods, and S. L. Eddins, Digital Image Processing Using MATLAB, Dorling Kindersley, New Delhi, India, 2004.

[19] R. C. Gonzalez and P. Wintz, Digital Image Processing, Addison- Wesley, Reading, Mass, USA, 1987.

[20] M. Roumi, Implementing texture feature algorithms on FPGA [M.S. thesis], Department of Computer Engineering, Delft University of Technology, Mekelweg, The Netherlands, 2009.

[21] F. Albregtsen, Statistical Texture Measures Computed from Gray Level Coocurrence Matrices, Image Processing Laboratory, Department of Informatics, University of Oslo, 2008.

[22] A. Rosenfeld, "Image analysis and computer vision: 1988," Computer Vision, Graphics and Image Processing, vol. 46, no. 2, pp. 196-250, 1989.

[23] J. Flusser, T. Suk, and B. Zitova, Moments and Moment Invariants in Pattern Recognition, John Wiley \& Sons, Chichester, UK, 2009.

[24] R. Mukundan and K. R. Ramakrishnan, Moment Functions in Image Analysis: Theory and Applications, World Scientific, Singapore, 1998.

[25] K. M. Hosny, "Refined translation and scale Legendre moment invariants," Pattern Recognition Letters, vol. 31, no. 7, pp. 533$538,2010$.

[26] K. M. Hosny, "Exact Legendre moment computation for gray level images," Pattern Recognition, vol. 40, no. 12, pp. 35973605, 2007. 

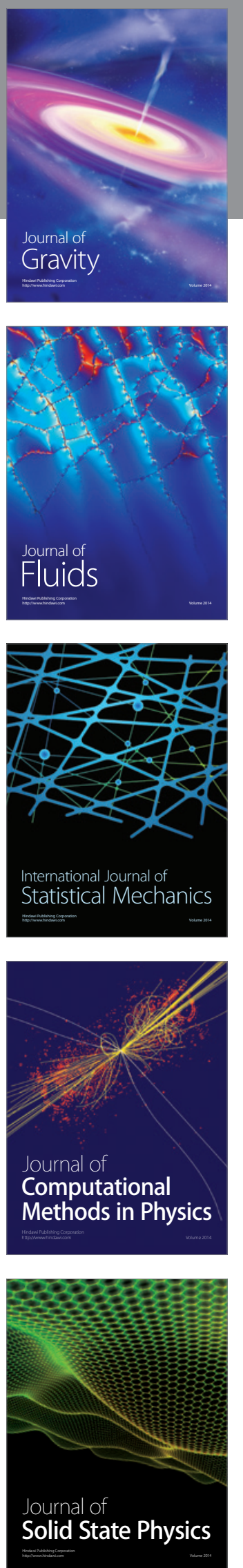
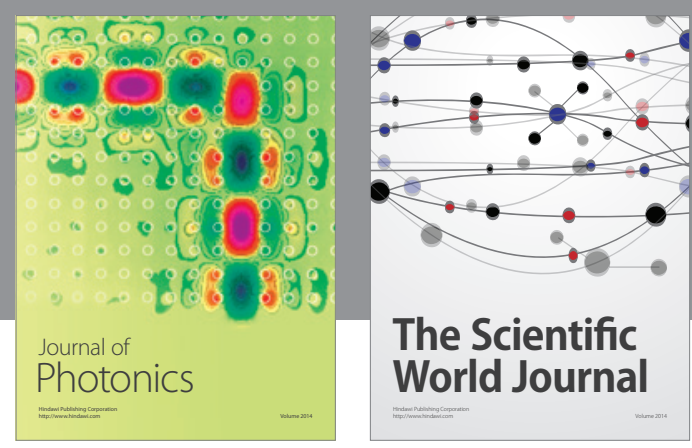

The Scientific World Journal

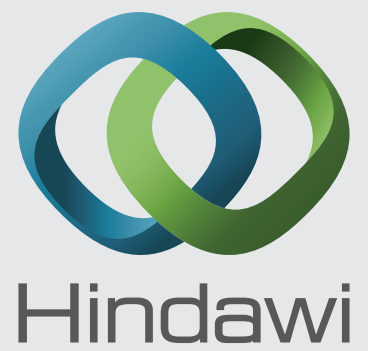

Submit your manuscripts at http://www.hindawi.com
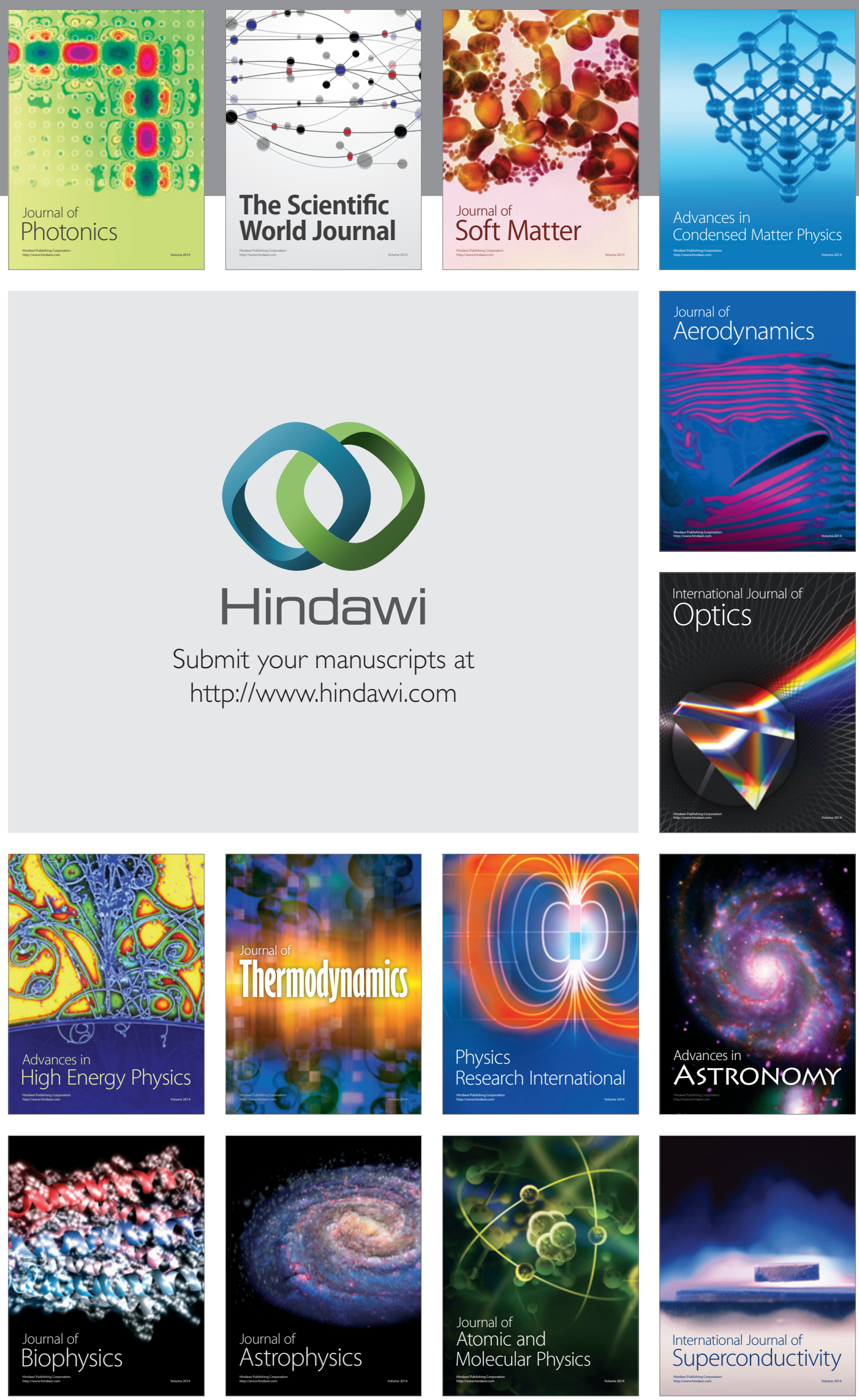
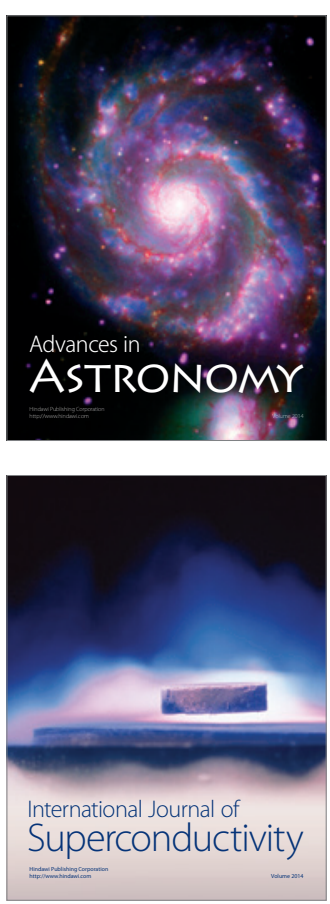